\title{
Pulmonary tuberculosis as a risk factor for chronic obstructive pulmonary disease: a systematic review and meta-analysis
}

\author{
Huanhuan Fan ${ }^{1 \#}$, Fan Wu ${ }^{2 \#}$, Jing Liu ${ }^{3 \#}$, Weifeng Zeng ${ }^{4}$, Silan Zheng ${ }^{3}$, Heshen Tian ${ }^{2}$, Haiqing Li $^{5}$, \\ Huajing Yang ${ }^{2}$, Zihui Wang ${ }^{2}$, Zhishan Deng ${ }^{2}$, Jieqi Peng ${ }^{2}$, Youlan Zheng ${ }^{2}$, Shan Xiao ${ }^{2}$, Guoping Hu$^{5}$, \\ Yumin Zhou ${ }^{2}$, Pixin Ran $^{2}$
}

${ }^{1}$ The Third Clinical College, the Third Affiliated Hospital of Guangzhou Medical University, Guangzhou, China; ${ }^{2}$ State Key Laboratory of Respiratory Disease \& National Clinical Research Center for Respiratory Disease, Guangzhou Institute of Respiratory Health, the First Affiliated Hospital of Guangzhou Medical University, Guangzhou, China; ${ }^{3}$ The First Clinical College, the First Affiliated Hospital of Guangzhou Medical University, Guangzhou, China; ${ }^{4}$ School of Public Health, Guangzhou Medical University, Guangzhou, China; ${ }^{5}$ Department of Respiratory Medicine, the Third Affiliated Hospital of Guangzhou Medical University, Guangzhou, China

Contributions: (I) Conception and design: P Ran, Y Zhou, F Wu, H Fan; (II) Administrative support: P Ran; (III) Provision of study materials or patients: None; (IV) Collection and assembly of data: H Fan, F Wu, J Liu, W Zeng, S Zheng; (V) Data analysis and interpretation: P Ran, Y Zhou, F Wu, H Fan, J Liu; (VI) Manuscript writing: All authors; (VII) Final approval of manuscript: All authors.

\#These authors contributed equally to this work.

Correspondence to: Pixin Ran. State Key Laboratory of Respiratory Disease \& National Clinical Research Center for Respiratory Disease, Guangzhou Institute of Respiratory Health, the First Affiliated Hospital of Guangzhou Medical University, 151 Yanjiang Xi Road, Guangzhou 510120, China. Email: pxran@gzhmu.edu.cn.

Background: Prior pulmonary tuberculosis (TB) can cause permanent changes in lung anatomy and is associated with lung function loss. However, it remains unclear whether pulmonary function impairment owing to TB is associated with airflow obstruction, the hallmark of chronic obstructive pulmonary disease (COPD). The aim of this systematic review and meta-analysis was to assess the association and quantify the magnitudes of association between pulmonary TB and COPD, and to evaluate the prevalence of COPD in patients with prior pulmonary TB.

Methods: We searched the PubMed, Embase, and Web of Science databases for studies published from inception to January 1, 2020. Pooled effect sizes were calculated according to a random effects model or fixed effect model depending on heterogeneity. Specific subgroups (different diagnostic criteria, smoking status, income level) were examined.

Results: A total of 23 articles were included in this study. Compared with controls, patients with pulmonary TB had an odds ratios (ORs) of 2.59 [95\% confidence interval (CI): 2.12-3.15; $\mathrm{P}<0.001]$ for developing COPD. In jackknife sensitivity analyses, the increased risk of prior pulmonary TB remained consistent for COPD; when the meta-analysis was repeated and one study was omitted each time, the ORs and corresponding 95\% CIs were greater than 2. Funnel plots of ORs with Egger's linear regression ( $\mathrm{t}=2.00$, $\mathrm{P}=0.058)$ and Begg's rank correlation $(\mathrm{Z}=0.75, \mathrm{P}=0.455)$ showing no significant publication bias. Subgroup analysis showed that the same conclusion was still present in never smokers (ORs 2.41; 95\% CI: 1.74-3.32; $\mathrm{P}<0.001$ ), patients with pulmonary TB diagnosed using chest $\mathrm{X}$-ray (ORs 2.47; 95\% CI: 1.23-4.97; $\mathrm{P}<0.001$ ), and low- and middle-income country (LMIC) settings (ORs 2.70; 95\% CI: 2.08-3.51; $\mathrm{P}<0.001$ ). The pooled prevalence of COPD in patients with prior pulmonary TB was $21 \%$ (95\% CI: $16-25 \%$; $<0.001$ ).

Conclusions: Individuals with prior pulmonary TB have an increased risk and high prevalence of COPD. Future studies identifying the underlying mechanisms for TB-associated COPD and therapeutic strategies are required.

^ ORCID: 0000-0003-0720-4674. 
Keywords: Chronic obstructive pulmonary disease (COPD); pulmonary tuberculosis (pulmonary TB); risk factor; meta-analysis

Submitted Jun 10, 2020. Accepted for publication Nov 29, 2020.

doi: $10.21037 / \mathrm{atm}-20-4576$

View this article at: http://dx.doi.org/10.21037/atm-20-4576

\section{Introduction}

Chronic obstructive pulmonary disease (COPD) is an umbrella term for various clinical entities, and the airflow obstruction cannot be fully reversible due to various reasons (1). COPD led to 3.2 million deaths in 2017 and will be the third leading cause of death globally by 2030 $(2,3)$. The most conventional risk factor for COPD is smoking, but smoking certainly is not the only factor (4). Over $90 \%$ of COPD-related morbidity and mortality occurs in low- and middle-income country (LMIC) settings where a significant proportion of those with COPD are never smokers $(5,6)$. Thus, the current understanding of risk factors for COPD is incomplete in many respects.

Tuberculosis (TB) is the leading infectious disease worldwide, responsible for over 10 million new cases and 1.5 million deaths (7). The treatment success rate for TB is increasing; nevertheless, prior pulmonary TB can cause permanent damage to lung anatomy and is associated with lung function loss (8). It is unclear whether pulmonary function impairment owing to TB is associated with airflow obstruction, the hallmark of COPD.

One previous systematic review published in 2013 had only summarized the risk of COPD in patients with prior pulmonary TB but this systematic review was not quantitative analyses (9). Another previous systematic review published in 2015 quantitative analyzed the relationship between prior pulmonary TB and COPD but this systematic review included the study of bronchiectasis in quantitative synthesis (10). Since 2015, many new studies have looked at the association between pulmonary TB and COPD. Therefore, current evidence and comprehensive quantitative analysis of prior pulmonary TB as a risk factor for COPD are needed to provide clinicians and researchers with the most up-to-date information. At the same time, pooling the effects of individual studies in meta-analysis may improve the statistical power and help to identify modest risk differences among study groups. Moreover, our study was able to perform subgroup meta-analyses and generate pooled ORs for various definitions of COPD, definitions of prior pulmonary TB, income level, and smoking status. With this in mind, we performed a systematic review and metaanalysis to evaluate the epidemiological links and magnitude of the association between COPD and prior pulmonary TB. We present the following article in accordance with the PRISMA reporting checklist (available at http://dx.doi. org/10.21037/atm-20-4576) (11).

\section{Methods}

The study protocol was registered with the International Prospective Register of Systematic Reviews (registration number CRD42020153304, https://www.crd.york.ac.uk/ PROSPERO/).

\section{Literature search}

Two investigators (FH and JL) independently searched the Embase, Web of Science and PubMed databases for studies published from inception to January 1, 2020, with no language restrictions. The search terms and related variants used included "COPD", "chronic obstructive pulmonary disease", "tuberculosis", and "TB". Further details of the search strategy are presented in Appendix 1. The terms were chosen by two investigators (FH and JL) and by checking keywords in other articles and reviews on similar topics. The references from other related articles and the selected papers were manually reviewed to identify potential studies of interest.

\section{Study selection}

Two investigators (HF and JL) independently reviewed all potentially relevant manuscripts. Disagreements or uncertainties were resolved by a third investigator (FW). The initial investigation included reviews of article titles and abstracts. Articles were mainly excluded owing to nonCOPD comorbidities and unextractable data. The secondary investigation included full-text review, and selection of articles based on inclusion and exclusion criteria. The studies 
included in this systematic review and meta-analysis met the following inclusion criteria: (I) case-control study, crosssectional study, or cohort study design; (II) comparison of at least two groups (COPD and control group, or prior pulmonary TB and control group); (III) reporting odds ratio (OR) to estimate the association between COPD and TB, with a corresponding $95 \%$ confidence interval (CI), or enough data to calculate these; (IV) independent of other studies. Studies with the same data set as published studies were not considered independent. We selected observational studies that included a specific definition for COPD and TB. The preferred definition of COPD was according to the Global Initiative for Chronic Obstructive Lung Disease (GOLD) criteria [post-bronchodilator forced expiratory volume in 1 second $\left(\mathrm{FEV}_{1}\right) /$ forced vital capacity $\left.(\mathrm{FVC})<0.7\right]$. Articles using pre-bronchodilator $\mathrm{FEV}_{1} / \mathrm{FVC}<0.7, \mathrm{FEV}_{1} / \mathrm{FVC}<$ the lower limit of normal (LLN), and chronic bronchitis (CB) were included, although some cases were not characterized by airflow obstruction that is not completely reversible. CB was clinically diagnosed as chronic cough or phlegm on most days for $>3$ months per year for at least 2 consecutive years. The definitions for TB included self-reported TB or TB lesions on chest $\mathrm{X}$-ray.

\section{Data extraction}

Two investigators (HF and JL) independently evaluated the quality of all identified studies and extracted and entered the data. These authors then independently verified the quality of the identified studies and validity of the extracted data. Differences were resolved in discussion or if necessary, by a third investigator (FW). We divided income level according to World Bank standards (12). The extracted data were recorded as follows: first author, year of publication, location, income level, population, study design (cross-sectional study, case-control study, or cohort study), characteristics of study participants (sample size, age, definition of prior pulmonary TB, and diagnosis criteria of COPD), proportion of patients with COPD who had TB, and $\mathrm{OR}$ and $95 \% \mathrm{CI}$ of the main outcome.

\section{Quality assessment of included studies}

Two investigators (HF and JL) independently assessed the quality of the included studies using a 22 -point quality assessment tool based on the Strengthening the Reporting of Observational Studies in Epidemiology (STROBE) score, which is a quality measure for observational studies (13). The highest possible score is 22 points, as shown in Table 1.

\section{Statistical analysis}

The primary outcome of this meta-analysis was the OR for COPD in patients with prior pulmonary TB compared with the population without pulmonary $\mathrm{TB}$, and the pooled prevalence of COPD in individuals with prior pulmonary TB. Subgroup analyses were performed with stratification according to definition of COPD, definition of prior pulmonary $\mathrm{TB}$, income level, and smoking status. Heterogeneity within the pooled studies was evaluated using the $\mathrm{I}^{2}$ statistic (significant if $\mathrm{I}^{2} \geq 50 \%$ ); depending on whether there was heterogeneity between studies, a fixed effects model or random effects model was used (37). Publication bias was evaluated using funnel plots, Begg's tests, and Egger's tests (38). We also conducted a jackknife sensitivity analysis to limit the impact of outliers or extreme observations. All statistical analyses were conducted using Stata/SE version 15.1 (StataCorp LLC, College Station, TX, USA), with $\mathrm{P}$ values less than 0.05 considered statistically significant.

\section{Results}

\section{Literature search and general description of included studies}

Figure 1 shows the literature screening flow chart, including the key words used in the search strategies, the number of papers identified in the databases (PubMed, Embase, and Web of Science), and the number of excluded studies and reason for exclusion. Of 5,174 abstracts identified during the search, 107 were selected for full-text review; 5,067 were excluded because the topic of this review was not evaluated. After reading the full text, an additional 84 articles were excluded for the following reasons: narrative review article; did not include patients with COPD who had prior pulmonary TB; duplicated/replicated data from the same study population; and outcome of interest not reported. Finally, 23 studies were included in the qualitative synthesis (meta-analysis) (14-36).

The characteristics of the included studies are presented in Table 1. In total, 36641 participants with COPD and 491538 participants without COPD were included in 22 studies; one study did not report these data (35). The mean age of participants was 52.2 years in 19 included 


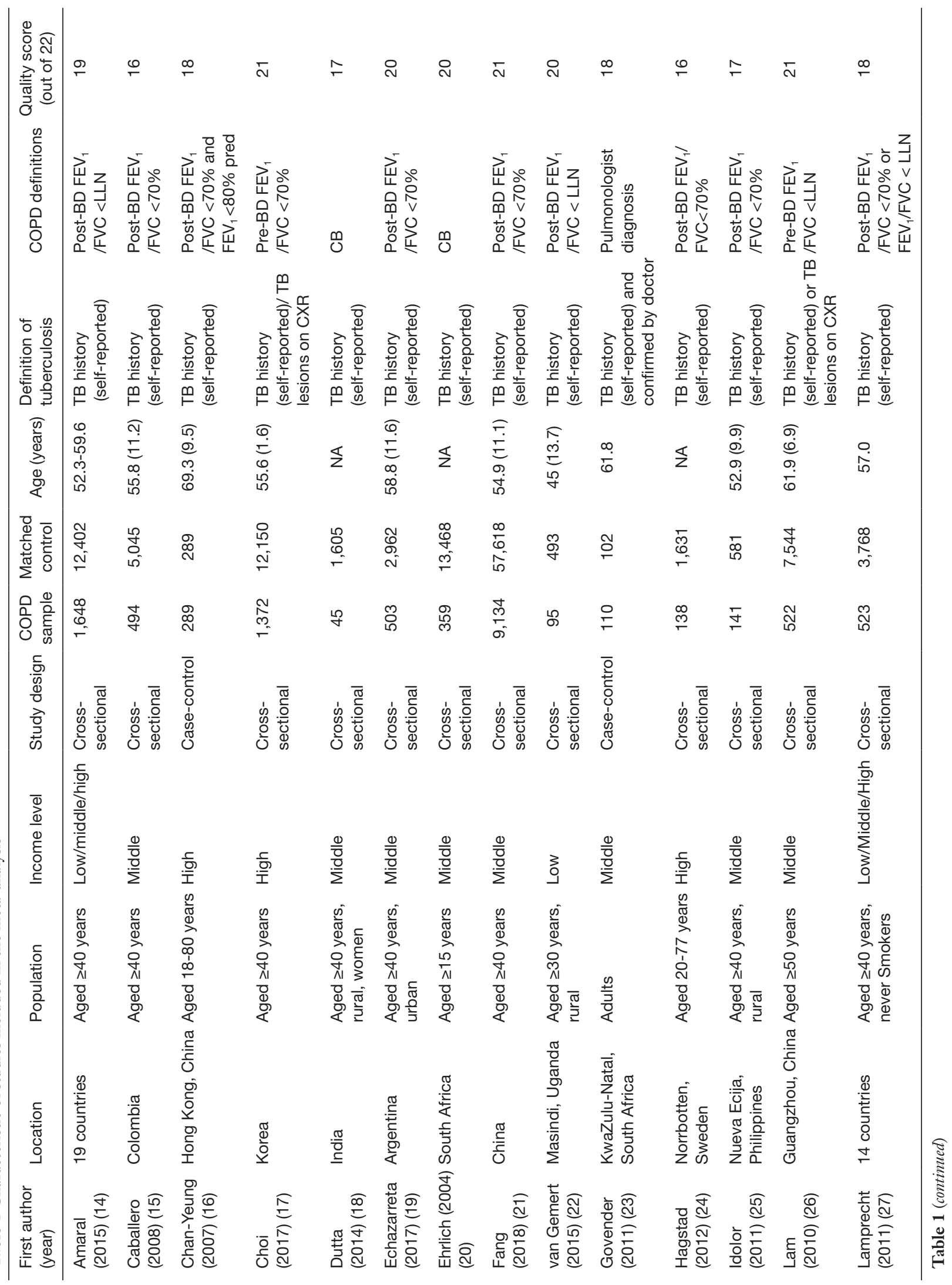




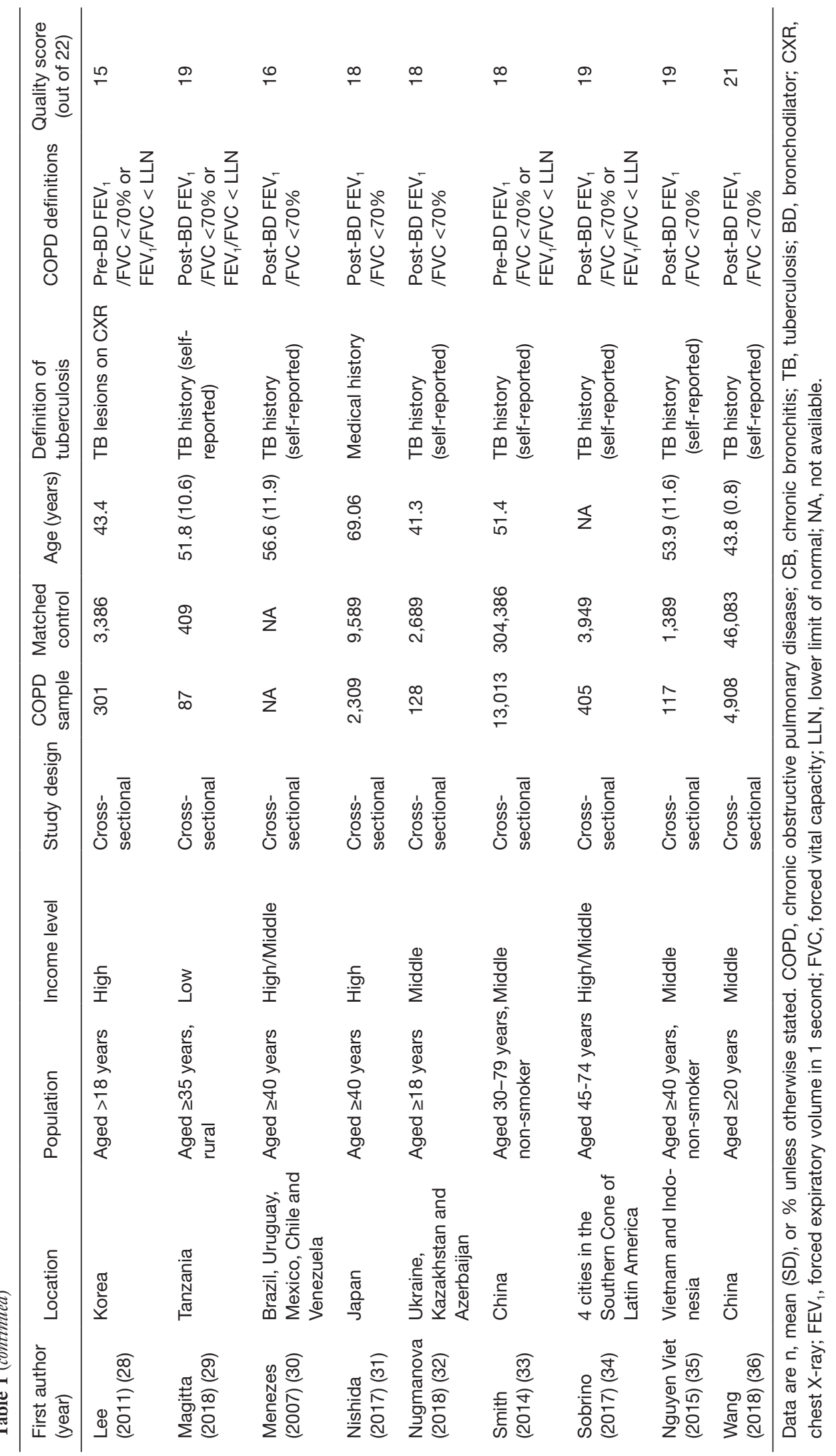




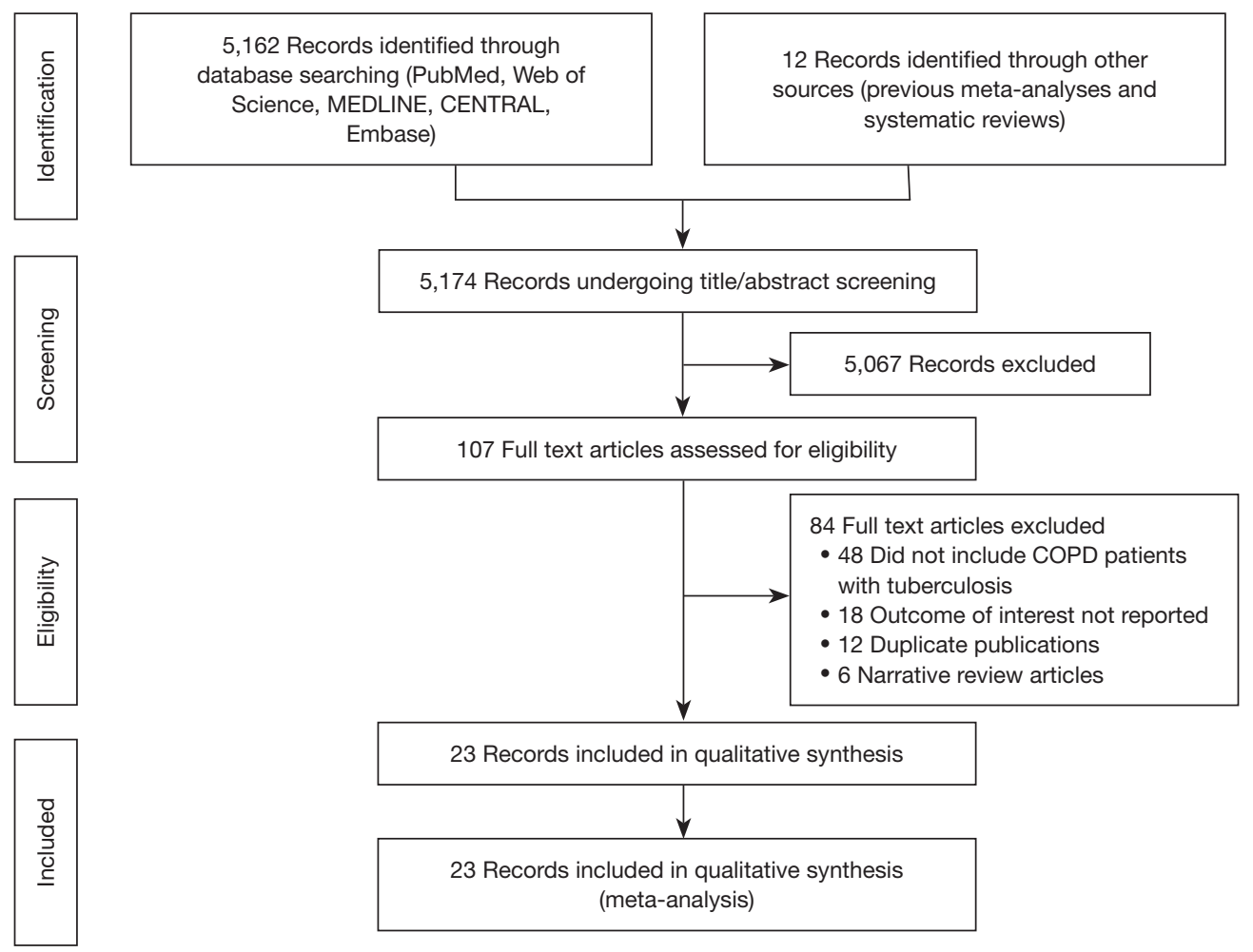

Figure 1 Preferred Reporting Items for Systematic Reviews and Meta-Analysis (PRISMA) flow diagram.

studies; 4 studies did not report these data $(18,20,24,34)$. According to the World Bank criteria for income levels, 5 studies were in high-income country settings and 14 studies were in LMIC settings. Data for two studies came from the Burden of Obstructive Lung Disease (BOLD) study; however, an article by Lamprecht et al. (27) provided information on the association between TB and COPD in never smokers, which was not provided by Amaral et al. (14). Therefore, the article by Lamprecht et al. was excluded from the primary meta-analysis but was used in subgroup analysis (27). This review included 13 studies with postbronchodilator $\mathrm{FEV}_{1} / \mathrm{FVC}<0.7$ as the main definition of COPD, 5 studies with pre-bronchodilator $\mathrm{FEV}_{1} / \mathrm{FVC}<0.7$ as the main definition of COPD, and 5 studies with $\mathrm{FEV}_{1}$ $/ \mathrm{FVC}<\mathrm{LLN}$ as the main definition of COPD.

All studies were published between 2004 and 2018. The methodological quality of the included studies was all satisfactory, with STROBE 22-point quality assessment scores between 15 to 21 .

\section{Association between TB and COPD}

Twenty-two studies examined the association between TB and COPD. Compared with patients who did not have a history of $\mathrm{TB}$, the pooled analysis found a significant elevation in risk of COPD for those with a history of TB (OR 2.59; 95\% CI: 2.12-3.15; $\mathrm{P}<0.001)$ with significant between-study heterogeneity $\left(\mathrm{I}^{2}=78.8 \%\right.$; $\mathrm{Tau}^{2}=0.144$; $\mathrm{P}<0.001$ ) (Figure 2). Nineteen studies adjusted for multiple confounding factors. Associations of COPD with TB exposure were not changed by adjusting for confounding. The pooled adjusted OR showed that TB was a significant risk factor for COPD (OR 2.53; 95\% CI: 2.04-3.13; $\mathrm{P}<0.001 ; \mathrm{I}^{2}=83.0 \% ; \mathrm{Tau}^{2}=0.148 ; \mathrm{P}<0.001$ ) (Figure 3).

In the jackknife sensitivity analyses, the increased risk with prior pulmonary TB remained consistent for COPD; when the meta-analysis was repeated and one study was omitted each time, the OR and corresponding 95\% CI were greater than 2 (Figure 4). Funnel plots of ORs were visually symmetric (Figure 5), with Begg's rank correlation $(\mathrm{Z}=0.75$, $\mathrm{P}=0.455)$ and Egger's linear regression $(\mathrm{t}=2.00, \mathrm{P}=0.058)$ showing no obvious publication bias (Figure S1).

\section{Subgroup analysis}

A total of seven studies analyzed never smokers. The 


\begin{tabular}{|c|c|c|}
\hline $\begin{array}{l}\text { Study } \\
\text { ID }\end{array}$ & OR $(95 \% \mathrm{CI})$ & $\begin{array}{l}\% \\
\text { Weight }\end{array}$ \\
\hline Amaral (2015) & $2.51(1.83,3.42)$ & 5.99 \\
\hline Caballero (2008) & $2.94(1.58,5.49)$ & 4.14 \\
\hline Chan-Yeung (2007) & $1.52(0.45,5.19)$ & 1.90 \\
\hline Choi (2017) & $4.47(3.07,6.51)$ & 5.61 \\
\hline Dutta (2014) & $9.08(2.47,34.03)$ & 1.71 \\
\hline Echazarreta (2017) & $3.30(1.43,7.62)$ & 3.11 \\
\hline Ehrilich-men (2004) & $4.90(2.60,9.10)$ & 4.12 \\
\hline Ehrilich-women (2004) & $6.60(3.70,11.70)$ & 4.41 \\
\hline Fang (2018) & $1.73(1.33,2.23)$ & 6.29 \\
\hline Gemert (2015) & $1.87(0.50,7.06)$ & 1.69 \\
\hline Govender (2011) & $9.14(2.06,40.64)$ & 1.40 \\
\hline Hagstad-never smoker (2012) & $1.58(0.20,12.36)$ & 0.81 \\
\hline Hagstad-ever smoker (2012) & $3.17(1.30,7.72)$ & 2.89 \\
\hline Idolor (2011) & $6.31(2.67,15.00)$ & 3.00 \\
\hline Lam (2010) & $1.37(1.13,1.67)$ & 6.59 \\
\hline Lee (2011) & $2.56(1.84,3.56)$ & 5.89 \\
\hline Magitta (2018) & $5.93(2.05,17.18)$ & 2.32 \\
\hline Menezes (2007) & $2.33(1.50,3.62)$ & 5.22 \\
\hline Nishida (2017) & $1.72(1.39,2.11)$ & 6.53 \\
\hline Nugmanova (2018) & $1.77(0.54,5.82)$ & 1.98 \\
\hline Smith-women (2014) & $2.36(2.06,2.71)$ & 6.81 \\
\hline Smith-men (2014) & $1.81(1.40,2.34)$ & 6.29 \\
\hline Sobrino (2017) & $3.30(1.40,7.80)$ & 3.02 \\
\hline Viet (2015) & $3.65(1.91,6.97)$ & 4.01 \\
\hline Wang (2018) & $0.55(0.30,1.00)$ & 4.26 \\
\hline Overall $($ I-squared $=78.8 \%, p=0.000)$ & $2.59(2.12,3.15)$ & 100.00 \\
\hline NOTE: Weights are from random effects analysis & & \\
\hline
\end{tabular}

Figure 2 Forest plot of ORs for COPD in prior pulmonary tuberculosis. OR, odds ratio; COPD, chronic obstructive pulmonary disease.

pooled OR showed that TB was a significant risk factor for COPD among never smokers (OR 2.41; 95\% CI: 1.74-3.32; $\mathrm{P}<0.001 ; \mathrm{I}^{2}=73.0 \% ; \mathrm{Tau}^{2}=0.130 ; \mathrm{P}<0.001$ ) (Table 2).

The different definitions of $\mathrm{TB}$ did not affect the results. The pooled OR showed that both self-reported TB and TB diagnosed with lesions on chest $\mathrm{X}$-ray were significant risk factors for COPD (defined using self-report: OR 2.61; 95\% CI: 2.13-3.21; $\mathrm{P}<0.001 ; \mathrm{I}^{2}=75.4 \% ; \mathrm{Tau}^{2}=0.142 ; \mathrm{P}<0.001$, and defined using chest $\mathrm{X}$-ray: OR 2.47; 95\% CI: 1.23-4.97; $\mathrm{P}<0.001 ; \mathrm{I}^{2}=94.1 \% ; \mathrm{Tau}^{2}=0.358 ; \mathrm{P}<0.001$ ) (Table 2).

According to the definition of COPD, patients were classified into three subgroups; the pooled results showed that prior pulmonary TB was a risk factor for COPD, even using different definitions of COPD (defined as prebronchodilator $\mathrm{FEV}_{1} / \mathrm{FVC}<0.7$ : $\mathrm{OR} 2.35$; $95 \% \mathrm{CI}$ : 1.82 3.04; $\mathrm{P}<0.001 ; \mathrm{I}^{2}=82.6 \%$; $\mathrm{Tau}^{2}=0.068 ; \mathrm{P}<0.001$, defined as post-bronchodilator $\mathrm{FEV}_{1} / \mathrm{FVC}<0.7$ : OR $2.31 ; 95 \% \mathrm{CI}$ : 1.68-3.18; $\mathrm{P}<0.001 ; \mathrm{I}^{2}=64.6 \%$; $\mathrm{Tau}^{2}=0.218 ; \mathrm{P}<0.001$, and defined as $\mathrm{FEV}_{1} / \mathrm{FVC}<\mathrm{LLN}$ : OR 2.32; 95\% CI: 1.50-3.57; $\mathrm{P}<0.001 ; \mathrm{I}^{2}=83.2 \% ; \mathrm{Tau}^{2}=0.165 ; \mathrm{P}<0.001$ ) (Table 2).

Using a random effects model, the pooled analysis showed that TB significantly increased the risk of developing COPD in both high-income and LMIC settings (high-income: OR 2.44; 95\% CI: 1.70-3.49; $\mathrm{P}<0.001 ; \mathrm{I}^{2}=71.5 \% ; \mathrm{Tau}^{2}=0.132 ; \mathrm{P}=0.002$, low- and middleincome: OR 2.70; 95\% CI: 2.08-3.51; $\mathrm{P}<0.001 ; \mathrm{I}^{2}=83.3 \%$; $\left.\mathrm{Tau}^{2}=0.188 ; \mathrm{P}<0.001\right)($ Table 2).

\section{Prevalence of COPD in prior pulmonary TB}

Sixteen studies including 1694 patients with TB reported a primary prevalence of COPD between $8 \%$ and $58 \%$. Metaanalysis demonstrated that the pooled prevalence of COPD in patients with prior pulmonary TB was $21 \%$ (95\% CI: $16-25 \%)$ with significant heterogeneity $\left(\mathrm{I}^{2}=96.8 \% ; \mathrm{P}<0.001\right)$ (Figure 6).

\section{Discussion}

The present meta-analysis was the first to quantitatively synthesize the risk factors and prevalence of COPD in patients with prior pulmonary TB. We found a nearly 


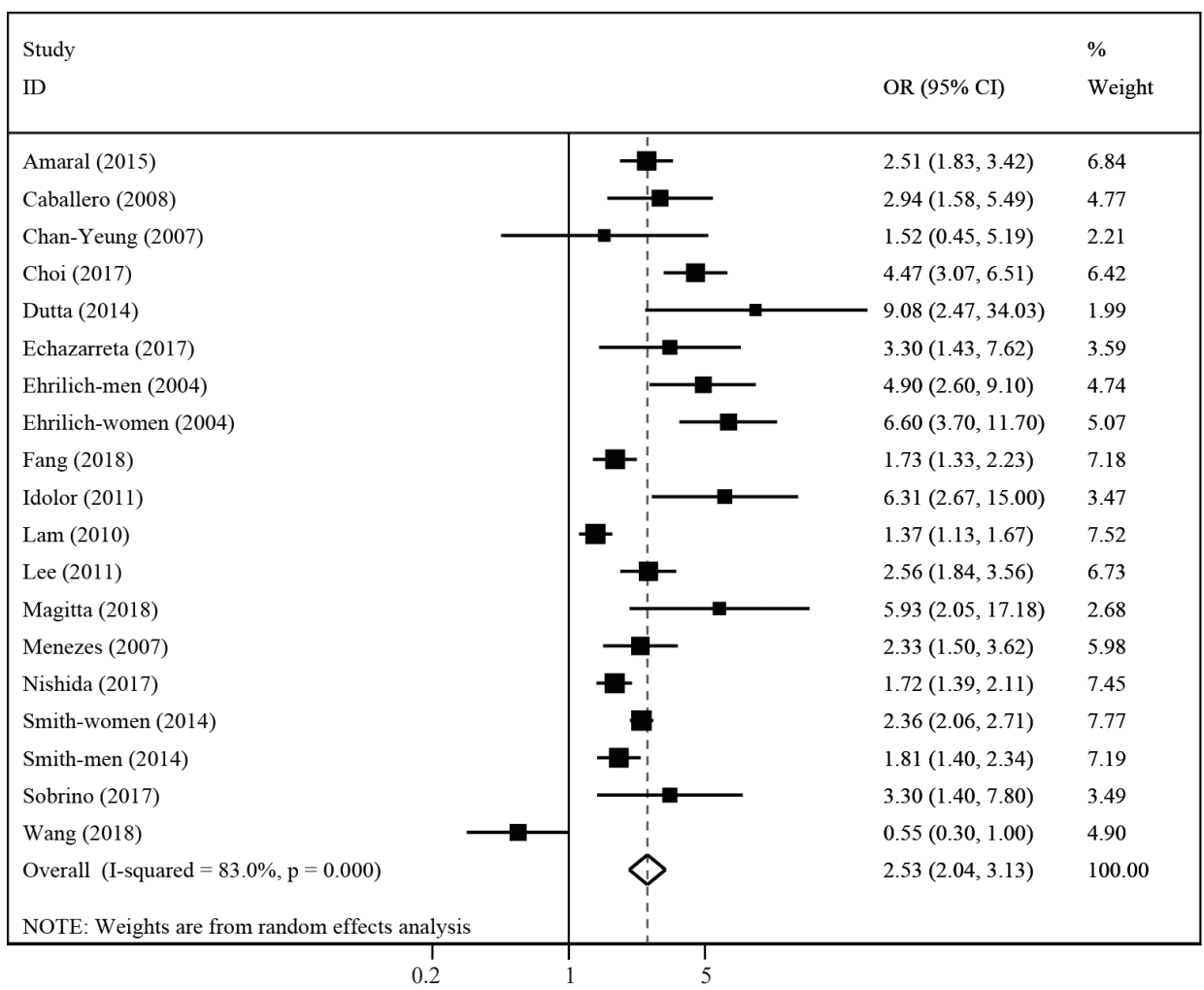

Figure 3 Forest plot of ORs for COPD in prior pulmonary tuberculosis adjusted by confounding factors. OR, odds ratio; COPD, chronic obstructive pulmonary disease.

2.6 times increased risk of COPD with prior pulmonary TB. In subgroup analyses, we found that this association was independent of smoking, income level, definition of COPD, definition of TB, and other confounding factors.

Our findings have important public health implications. On the one hand, more than $30 \%$ of the world's population may have latent $\mathrm{TB}$, and $5-20 \%$ of these individuals develop active TB at some point in their lifetime $(7,39)$. Patients with active TB usually develop sequelae in the lungs after treatment, which can lead to a decline in quality of life and disability. Our findings add to existing knowledge and support the association between prior pulmonary TB and COPD, which can provide a basis for clinicians to improve the early diagnosis of COPD, especially in areas where TB is prevalent.

On the other hand, inhaled corticosteroids (ICS) have been widely used in COPD treatment as these can reduce acute exacerbation in some patients with COPD, including those with a history of multiple or severe exacerbations despite appropriate maintenance bronchodilator use, patients with blood eosinophil counts $>300$ cells $/ \mu \mathrm{L}$, and those with a history of and/or concomitant asthma. However, ICS use can lead to the recurrence of pulmonary TB and increase the risk of TB among patients who do not have pulmonary TB. Therefore, ICS should not be used in patients with prior pulmonary TB (40). Considering the large number of patients with prior pulmonary $\mathrm{TB}$ and the high prevalence of COPD in LMICs, before prescribing ICS/long-acting $\beta 2$ agonists (LABA) or ICS/LABA/longacting muscarinic antagonists (LAMA) to patients with COPD, a chest radiograph or computed tomography scan should be performed to ensure patients with COPD do not have pulmonary TB.

The exact mechanism by which pulmonary TB leads to COPD is still unclear. Previous studies have found that accelerated emphysematous change caused by residual chronic or recurrent inflammation owing to $\mathrm{TB}$ and dysregulation of macrophages caused by latent intracellular infection may be linked to COPD (41-43). The primary function of macrophages in the lung is to kill bacteria or promote wound healing and resolution (44); it is well known that macrophages play a central role in the remodeling that 


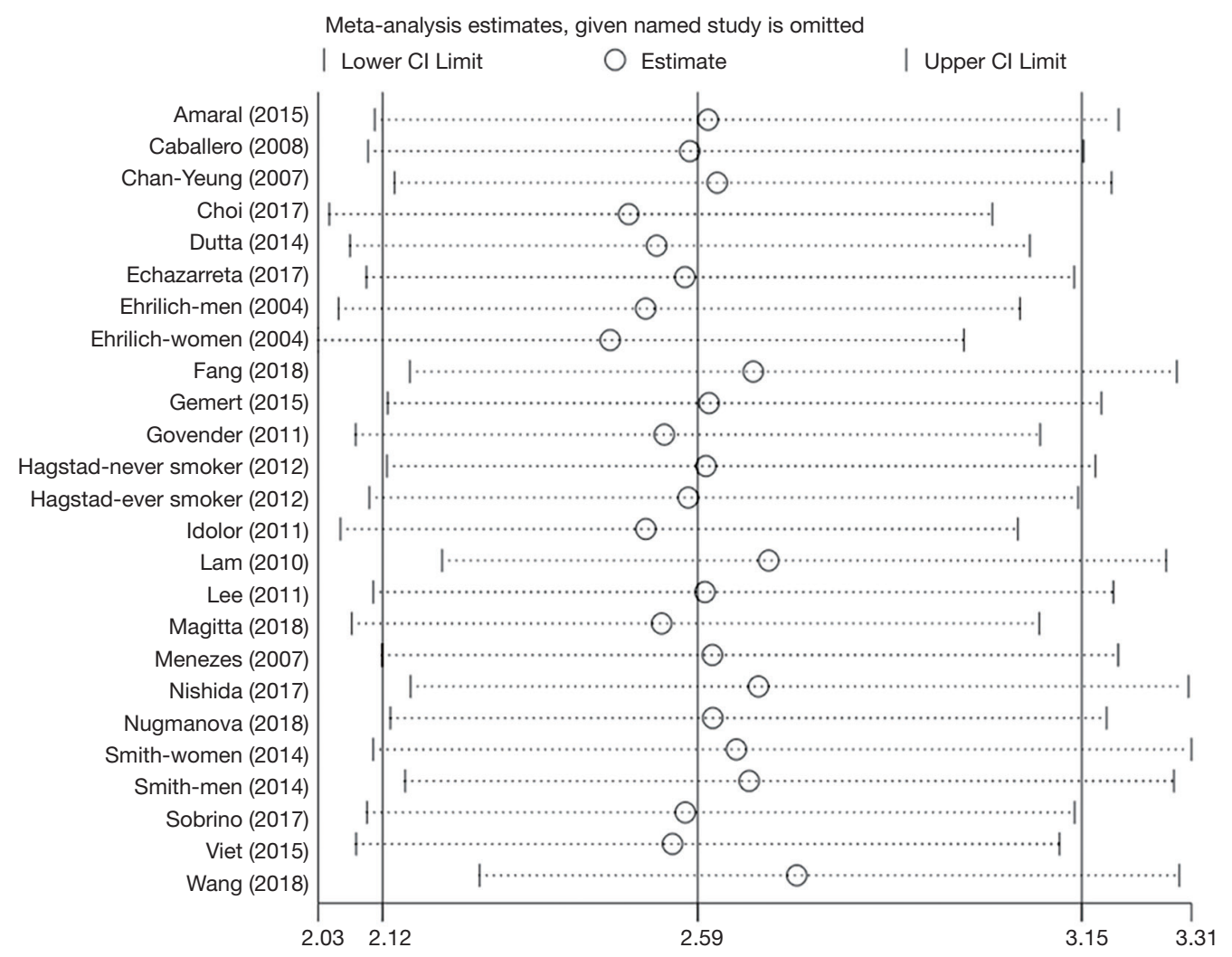

Figure 4 Jackknife sensitivity analyses. In the jackknife sensitivity analyses, the increased risk with prior pulmonary TB remained consistent for COPD; when the meta-analysis was repeated and one study was omitted each time, the OR and corresponding $95 \%$ CI were greater than 2. TB, tuberculosis; OR, odds ratio; COPD, chronic obstructive pulmonary disease; CI, confidence interval.

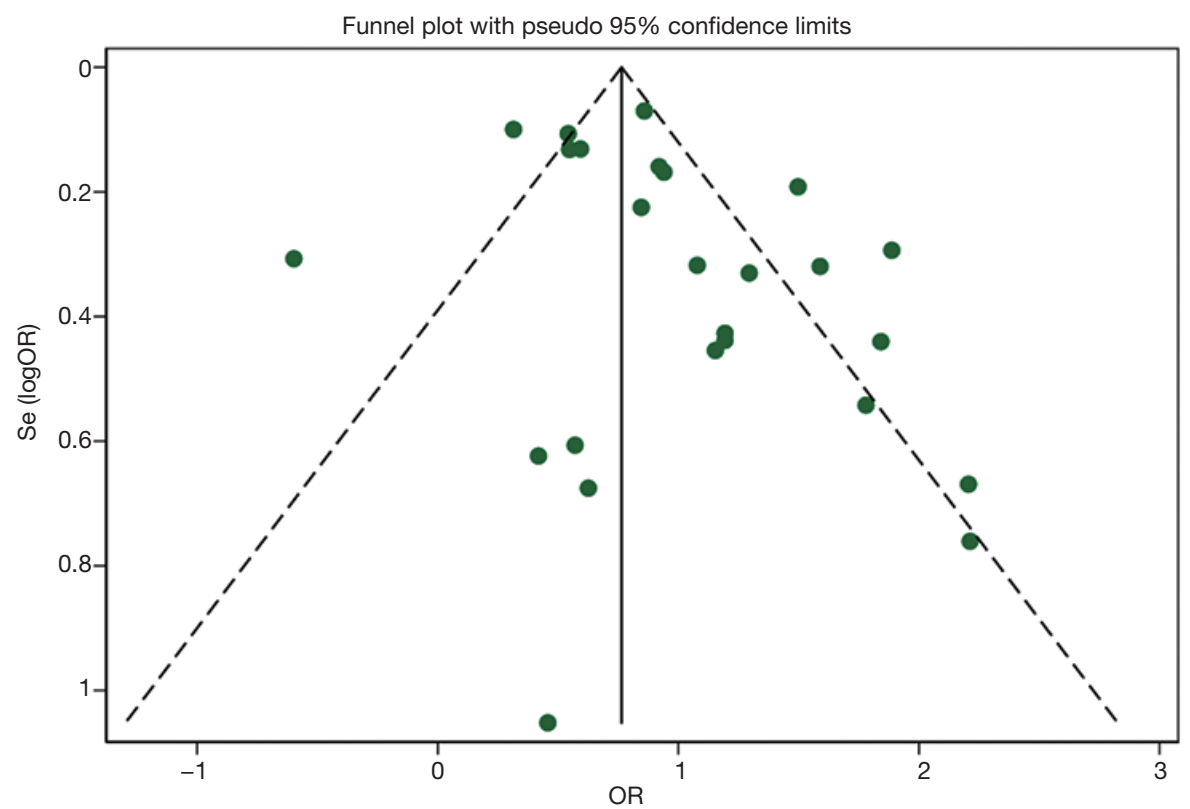

Figure 5 Funnel plots of ORs for COPD in prior pulmonary tuberculosis. Funnel plots of ORs were visually symmetric showing no obvious publication bias. OR, odds ratio; COPD, chronic obstructive pulmonary disease. 
Table 2 Association between prior pulmonary tuberculosis and COPD in subgroups

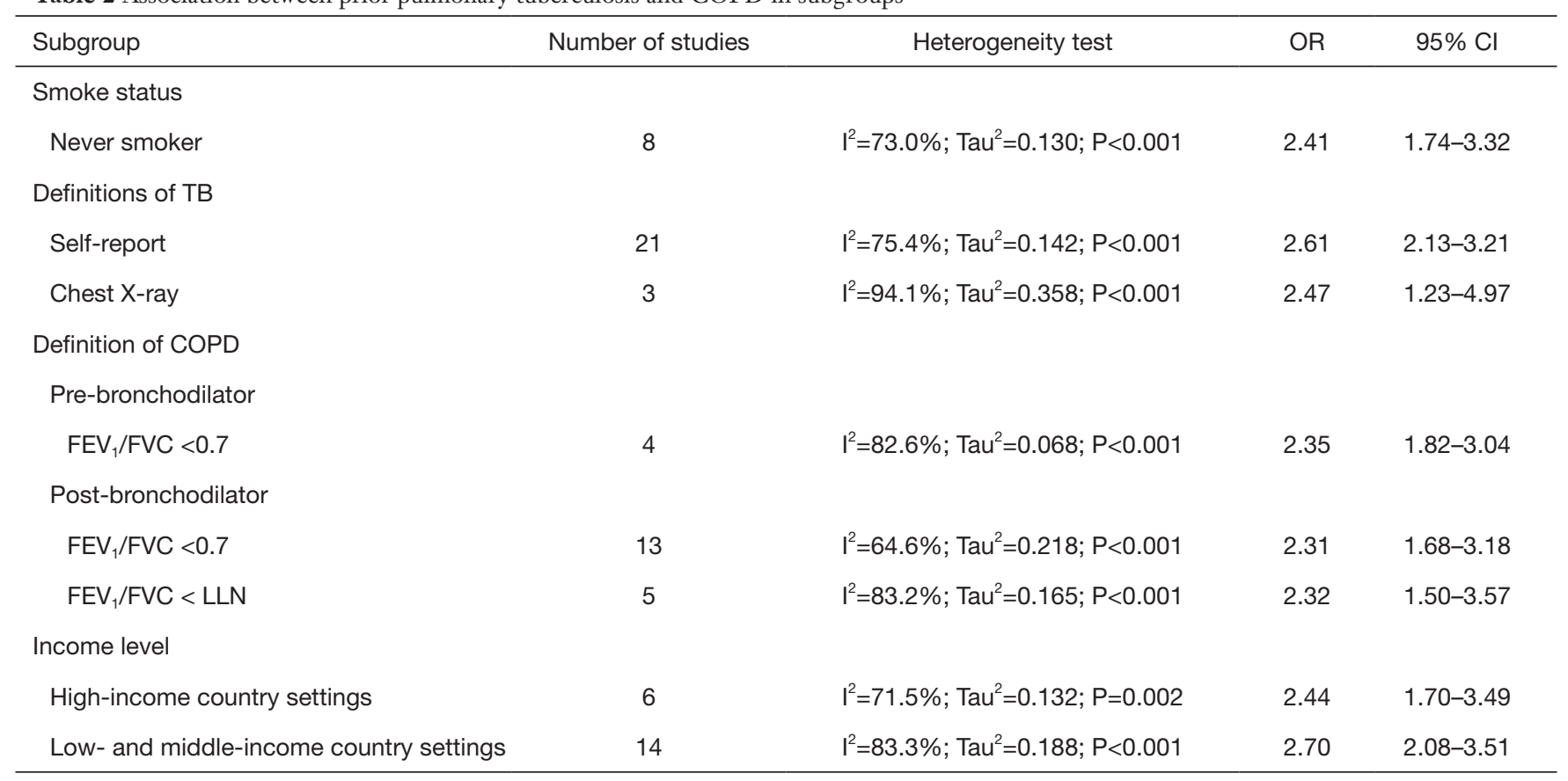

OR, odds ratio; $\mathrm{Cl}$, confidence interval; TB, tuberculosis; COPD, chronic obstructive pulmonary disease; FEV ${ }_{1}$, forced expiratory volume in 1 second; FVC, forced vital capacity; LLN, lower limit of normal.

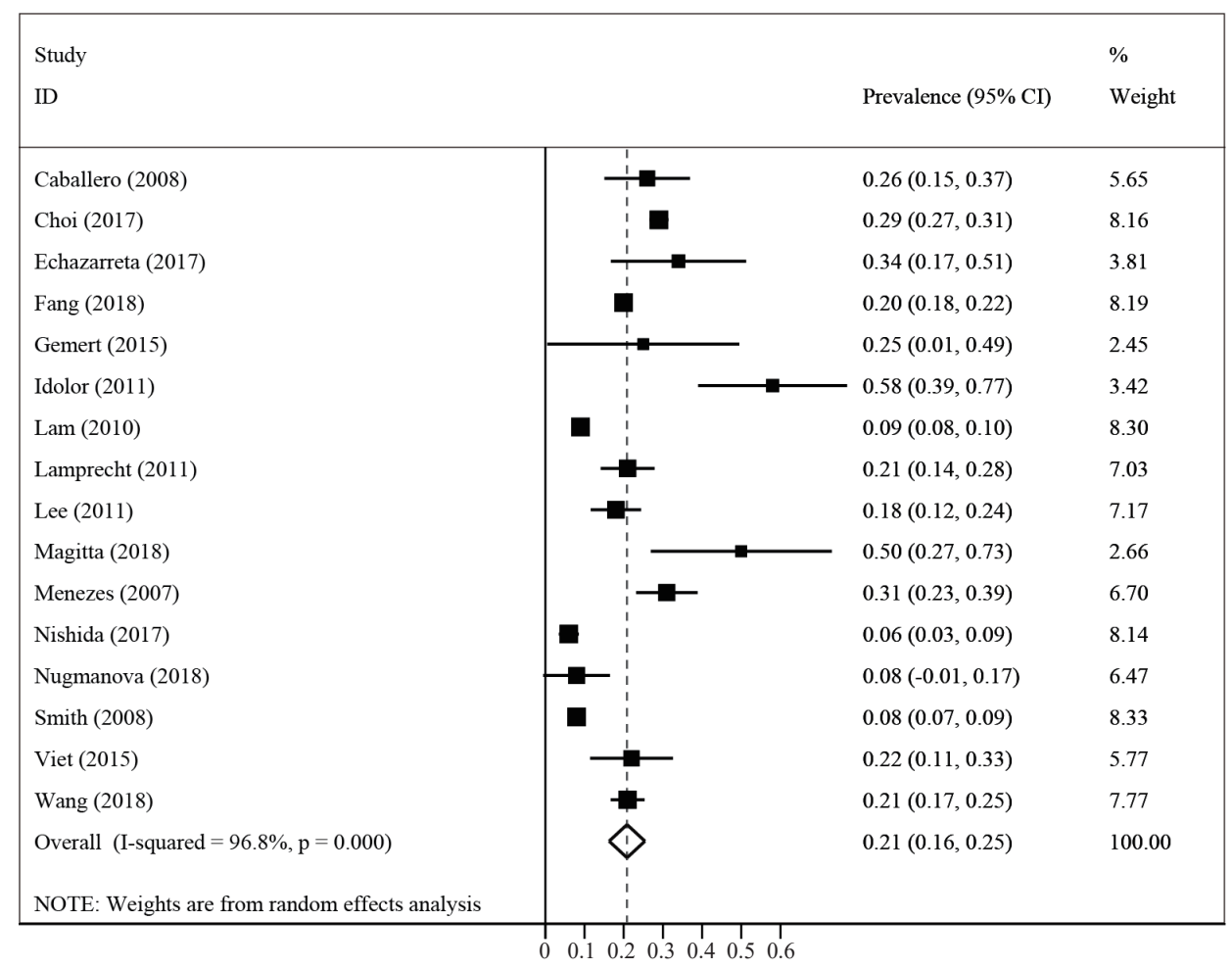

Figure 6 Forest plot of the prevalence of COPD in prior pulmonary tuberculosis. COPD, chronic obstructive pulmonary disease. 
leads to chronic airflow obstruction. Potential mycobacteria in lung macrophages may cause maintenance of lung inflammation and more severe airway changes $(42,45)$. Other mechanisms include loss of parenchymal tissue and scarring after $\mathrm{TB}$, and structural damage to the air passages and small airways, such as bronchiectasis owing to endobronchial involvement, bronchial stenosis, and bronchiolitis occlusion owing to peribronchial fibrosis (46). Another study showed that TB can lead to high activity of matrix metalloproteinases, leading to abnormal degradation of the extracellular matrix of the lung, which limits lung function (47). Future studies identifying the underlying immune mechanisms for $\mathrm{TB}$-associated $\mathrm{COPD}$, relative to the natural history of TB disease, are needed to help inform prognostic and therapeutic strategies for post-TB lung disease (6).

There are some potential limitations in this study that should be mentioned. First, a causal relationship between $\mathrm{TB}$ and COPD could not be determined because the current evidence was primarily from cross-sectional and case-control studies. Previous studies have found that ICS use is associated with an increased risk of TB in patients with obstructive lung diseases and TB may also be reactivated in individuals who take ICS (48). Some uncertainty remains regarding whether there is a causal relationship between prior pulmonary TB and COPD. However, patients with pulmonary TB have fairly marked destruction of the lung structure, and previous studies have found that patients with TB have faster lung function decline and meet the diagnostic criteria for COPD more than those without TB $(49,50)$. As a result, we believe that prior TB is a risk factor for COPD. Second, there was significant heterogeneity in the effect estimates across studies, resulting in reduced credibility of our research results. However, all effect estimates in the present metaanalysis for the association between prior pulmonary $\mathrm{TB}$ and COPD indicated a positive association. The jackknife sensitivity analysis did not change the conclusions, with one study omitted at a time, and there was no significant publication bias; thus, our conclusions remain credible. Third, the most common method used to define TB in the study was self-report, which may lead to recall bias and underreporting owing to the considerable stigma experienced by patients with TB (51). However, it is worth noting that $\mathrm{TB}$ remained a significant risk factor for COPD in the subgroup analysis of TB diagnosed using chest $\mathrm{X}$-ray.

In summary, we found that prior pulmonary $\mathrm{TB}$ was a significant risk factor for COPD and the prevalence of COPD among patients with TB was about 2.6 times higher than globally. A history of pulmonary TB is likely to be an important contributor to the burden of COPD in areas where pulmonary TB is endemic and among those who have never smoked. Nevertheless, large sample size longitudinal cohort studies using post-bronchodilator spirometry are still recommended to confirm these findings and to design effective strategies to limit this problem. As TB continues to spread in developing countries, the incidence of multidrug resistant diseases increases. With aging of the world's population, it is vital to improve our understanding of the mechanisms linking pulmonary TB to COPD and airflow obstruction, and to develop effective strategies to deal with this problem.

\section{Acknowledgments}

We thank Bijia Lin, Peiyu Huang, Shaodan Wei, Xiaopeng Lin (State Key Laboratory of Respiratory Disease \& National Clinical Research Center for Respiratory Disease, Guangzhou Institute of Respiratory Health, The First Affiliated Hospital of Guangzhou Medical University, Nanshan Medicine Innovation Institute of Guangdong Province), for their efforts in collecting the information and verification. We thank Nanshan Medical Development Foundation of Guangdong Province for its support. We thank Liwen Bianji, Edanz Group China (www.liwenbianji. $\mathrm{cn} / \mathrm{ac}$ ), for editing the English text of a draft of this manuscript.

Funding: This study was supported by the National Natural Science Foundation of China (81670040, 81970045), Local Innovative and Research Teams Project of Guangdong Pearl River Talents Program (2017BT01S155), and 135 Key Research and Development Program (2016YFC1304101).

\section{Footnote}

Reporting Checklist: The authors have completed the PRISMA reporting checklist. Available at http://dx.doi. org/10.21037/atm-20-4576

Conflicts of Interest: All authors have completed the ICMJE uniform disclosure form (available at http://dx.doi. org/10.21037/atm-20-4576). The authors have no conflicts of interest to declare.

Ethical Statement: The authors are accountable for all aspects 
of the work in ensuring that questions related to the accuracy or integrity of any part of the work are appropriately investigated and resolved. The study protocol was registered with the International Prospective Register of Systematic Reviews (registration number CRD42020153304, https:// www.crd.york.ac.uk/PROSPERO/).

Open Access Statement: This is an Open Access article distributed in accordance with the Creative Commons Attribution-NonCommercial-NoDerivs 4.0 International License (CC BY-NC-ND 4.0), which permits the noncommercial replication and distribution of the article with the strict proviso that no changes or edits are made and the original work is properly cited (including links to both the formal publication through the relevant DOI and the license). See: https://creativecommons.org/licenses/by-nc-nd/4.0/.

\section{References}

1. Celli BR, Wedzicha JA. Update on Clinical Aspects of Chronic Obstructive Pulmonary Disease. N Engl J Med 2019;381:1257-66.

2. Rabe KF, Watz H. Chronic obstructive pulmonary disease. Lancet 2017;389:1931-40.

3. GBD 2017 Causes of Death Collaborators. Global, regional, and national age-sex-specific mortality for 282 causes of death in 195 countries and territories, 1980-2017: a systematic analysis for the Global Burden of Disease Study 2017. Lancet 2018;392:1736-88.

4. Postma DS, Bush A, van den Berge M. Risk factors and early origins of chronic obstructive pulmonary disease. Lancet 2015;385:899-909.

5. Salvi SS, Barnes PJ. Chronic obstructive pulmonary disease in non-smokers. Lancet 2009;374:733-43.

6. Siddharthan T, Gupte A, Barnes PJ. COPD Endotypes in Low- and Middle-income Country Settings: Precision Medicine for All. Am J Respir Crit Care Med 2020.

7. World Health Organization. Global tuberculosis report 2019. Available online: https://www.who.int/tb/ publications/global_report/en/. Accessed May 242020.

8. Ravimohan S, Kornfeld H, Weissman D, et al. Tuberculosis and lung damage: from epidemiology to pathophysiology. Eur Respir Rev 2018;27.

9. Allwood BW, Myer L, Bateman ED. A systematic review of the association between pulmonary tuberculosis and the development of chronic airflow obstruction in adults. Respiration 2013;86:76-85.

10. Byrne AL, Marais BJ, Mitnick CD, et al. Tuberculosis and chronic respiratory disease: a systematic review. Int J Infect Dis 2015;32:138-46.

11. Moher D, Liberati A, Tetzlaff J, et al. Preferred reporting items for systematic reviews and metaanalyses: the PRISMA statement. PLoS Med 2009;6:e1000097.

12. the World Bank. The World by Income and Region. Available online: http://datatopics.worldbank.org/worlddevelopment-indicators/the-world-by-income-and-region. html. Accessed May 242020.

13. von Elm E, Altman DG, Egger M, et al. The Strengthening the Reporting of Observational Studies in Epidemiology (STROBE) statement: guidelines for reporting observational studies. Lancet 2007;370:1453-7.

14. Amaral AF, Coton S, Kato B, et al. Tuberculosis associates with both airflow obstruction and low lung function: BOLD results. Eur Respir J 2015;46:1104-12.

15. Caballero A, Torres-Duque CA, Jaramillo C, et al. Prevalence of COPD in five Colombian cities situated at low, medium, and high altitude (PREPOCOL study). Chest 2008;133:343-9.

16. Chan-Yeung M, Ho AS, Cheung AH, et al. Determinants of chronic obstructive pulmonary disease in Chinese patients in Hong Kong. Int J Tuberc Lung Dis 2007;11:502-7.

17. Choi CJ, Choi WS, Lee SY, et al. The Definition of Past Tuberculosis Affects the Magnitude of Association between Pulmonary Tuberculosis and Respiratory Dysfunction: Korea National Health and Nutrition Examination Survey, 2008-2012. J Korean Med Sci 2017;32:789-95.

18. Dutta S, Deshmukh PR. Prevalence and determinants of self-reported chronic bronchitis among women in rural Central India. Med J Armed Forces India 2015;71:48-52.

19. Echazarreta AL, Arias SJ, Del Olmo R, et al. Prevalence of COPD in 6 Urban Clusters in Argentina: The EPOC.AR Study. Arch Bronconeumol 2018;54:260-9.

20. Ehrlich RI, White N, Norman R, et al. Predictors of chronic bronchitis in South African adults. Int $\mathrm{J}$ Tuberc Lung Dis 2004;8:369-76.

21. Fang L, Gao P, Bao H, et al. Chronic obstructive pulmonary disease in China: a nationwide prevalence study. Lancet Respir Med 2018;6:421-30.

22. van Gemert F, Kirenga B, Chavannes N, et al. Prevalence of chronic obstructive pulmonary disease and associated risk factors in Uganda (FRESH AIR Uganda): a prospective cross-sectional observational study. Lancet Global Health 2015;3:e44-51. 
23. Govender N, Lalloo UG, Naidoo RN. Occupational exposures and chronic obstructive pulmonary disease: a hospital based case-control study. Thorax 2011;66:597-601.

24. Hagstad S, Ekerljung L, Lindberg A, et al. COPD among non-smokers - report from the obstructive lung disease in Northern Sweden (OLIN) studies. Respir Med 2012;106:980-8.

25. Idolor LF, TS DEG, Francisco NA, et al. Burden of obstructive lung disease in a rural setting in the Philippines. Respirology 2011;16:1111-8.

26. Lam KB, Jiang CQ, Jordan RE, et al. Prior TB, smoking, and airflow obstruction: a cross-sectional analysis of the Guangzhou Biobank Cohort Study. Chest 2010;137:593-600.

27. Lamprecht B, McBurnie MA, Vollmer WM, et al. COPD in never smokers: results from the populationbased burden of obstructive lung disease study. Chest 2011;139:752-63.

28. Lee SW, Kim YS, Kim DS, et al. The risk of obstructive lung disease by previous pulmonary tuberculosis in a country with intermediate burden of tuberculosis. J Korean Med Sci 2011;26:268-73.

29. Magitta NF, Walker RW, Apte KK, et al. Prevalence, risk factors and clinical correlates of COPD in a rural setting in Tanzania. Eur Respir J 2018;51:1700182.

30. Menezes AM, Hallal PC, Perez-Padilla R, et al. Tuberculosis and airflow obstruction: evidence from the PLATINO study in Latin America. Eur Respir J 2007;30:1180-5.

31. Nishida Y, Takahashi Y, Tezuka K, et al. A comprehensive analysis of association of medical history with airflow limitation: a cross-sectional study. Int J Chron Obstruct Pulmon Dis 2017;12:2363-71.

32. Nugmanova D, Feshchenko Y, Iashyna L, et al. The prevalence, burden and risk factors associated with chronic obstructive pulmonary disease in Commonwealth of Independent States (Ukraine, Kazakhstan and Azerbaijan): results of the CORE study. BMC Pulm Med 2018;18:26.

33. Smith M, Li L, Augustyn M, et al. Prevalence and correlates of airflow obstruction in approximately 317,000 never-smokers in China. Eur Respir J 2014;44:66-77.

34. Sobrino E, Irazola VE, Gutierrez L, et al. Estimating prevalence of chronic obstructive pulmonary disease in the Southern Cone of Latin America: how different spirometric criteria may affect disease burden and health policies. BMC Pulm Med 2017;17:187.

35. Nguyen Viet N, Yunus F, Nguyen Thi Phuong A, et al.
The prevalence and patient characteristics of chronic obstructive pulmonary disease in non-smokers in Vietnam and Indonesia: An observational survey. Respirology 2015;20:602-11.

36. Wang C, Xu J, Yang L, et al. Prevalence and risk factors of chronic obstructive pulmonary disease in China (the China Pulmonary Health $[\mathrm{CPH}]$ study): a national cross-sectional study. Lancet 2018;391:1706-17.

37. Higgins JP, Thompson SG, Deeks JJ, et al. Measuring inconsistency in meta-analyses. BMJ 2003;327:557-60.

38. Ioannidis JP, Trikalinos TA. The appropriateness of asymmetry tests for publication bias in meta-analyses: a large survey. CMAJ 2007;176:1091-6.

39. Selwyn PA, Hartel D, Lewis VA, et al. A prospective study of the risk of tuberculosis among intravenous drug users with human immunodeficiency virus infection. N Engl J Med 1989;320:545-50.

40. Agusti A, Fabbri LM, Singh D, et al. Inhaled corticosteroids in COPD: friend or foe? Eur Respir J 2018;52:1801219.

41. Chakrabarti B, Calverley PM, Davies PD. Tuberculosis and its incidence, special nature, and relationship with chronic obstructive pulmonary disease. Int J Chron Obstruct Pulmon Dis 2007;2:263-72.

42. Holloway RA, Donnelly LE. Immunopathogenesis of chronic obstructive pulmonary disease. Curr Opin Pulm Med 2013;19:95-102.

43. Jordan TS, Spencer EM, Davies P. Tuberculosis, bronchiectasis and chronic airflow obstruction. Respirology 2010;15:623-8.

44. Alber A, Howie SE, Wallace WA, et al. The role of macrophages in healing the wounded lung. Int J Exp Pathol 2012;93:243-51.

45. Mosser DM, Edwards JP. Exploring the full spectrum of macrophage activation. Nat Rev Immunol 2008;8:958-69.

46. Dheda $\mathrm{K}$, Booth $\mathrm{H}$, Huggett JF, et al. Lung remodeling in pulmonary tuberculosis. J Infect Dis 2005;192:1201-9.

47. Elkington PT, Friedland JS. Matrix metalloproteinases in destructive pulmonary pathology. Thorax 2006;61:259-66.

48. Castellana G, Castellana M, Castellana C, et al. Inhaled Corticosteroids And Risk Of Tuberculosis In Patients With Obstructive Lung Diseases: A Systematic Review And Meta-Analysis Of Non-randomized Studies. Int J Chron Obstruct Pulmon Dis 2019;14:2219-27.

49. Park HJ, Byun MK, Kim HJ, et al. History of pulmonary tuberculosis affects the severity and clinical outcomes of COPD. Respirology 2018;23:100-6. 
50. Jung JW, Choi JC, Shin JW, et al. Pulmonary Impairment in Tuberculosis Survivors: The Korean National Health and Nutrition Examination Survey 2008-2012. PLoS One 2015;10:e0141230.

Cite this article as: Fan $\mathrm{H}, \mathrm{Wu}$ F, Liu J, Zeng W, Zheng S, Tian H, Li H, Yang H, Wang Z, Deng Z, Peng J, Zheng Y, Xiao S, Hu G, Zhou Y, Ran P. Pulmonary tuberculosis as a risk factor for chronic obstructive pulmonary disease: a systematic review and meta-analysis. Ann Transl Med 2021;9(5):390. doi: 10.21037/atm-20-4576
51. Kelly P. Isolation and stigma: the experience of patients with active tuberculosis. J Community Health Nurs 1999;16:233-41. 


\section{Additional file1. Search and study selection strategy}

\#1 "COPD"

\#2 "obstructive lung disease"

\#3 "COPD" OR "obstructive lung disease"

\#4 "tuberculosis"

\#5 "TB"

\#6 "tuberculosis"[MeSH]

\#7 "tuberculosis" OR "TB" OR "tuberculosis"[MeSH]

\#8 ("COPD" OR "obstructive lung disease") AND ("tuberculosis" OR "TB" OR "tuberculosis"[MeSH])

\#9 "prevalence"

\#10 "prevalence"[MeSH]

\#11 "prevalence" OR "prevalence"[MeSH]

\#12 ("COPD" OR "obstructive lung disease") AND ("prevalence" OR "prevalence"[MeSH])

\#13 "risk factors"[Title/Abstract]

\#14 ("risk factors"[Title/Abstract]) AND ("COPD" OR "obstructive lung disease")

\#15 ("risk factors"[Title/Abstract]) OR ("prevalence" OR "prevalence"[MeSH]) OR ("tuberculosis" OR "TB" OR "tuberculosis"[MeSH])

\#16 (("risk factors"[Title/Abstract]) OR ("prevalence" OR "prevalence"[MeSH]) OR ("tuberculosis" OR "TB" OR "tuberculosis"[MeSH])) AND ("COPD" OR "obstructive lung disease")

\#17 "review"[Publication Type]

\#18 "Systematic Reviews as Topic"[MeSH]

\#19 "review"[Filter]

\#20 "Systematic Reviews as Topic"[MeSH] OR "review"[Publication Type] OR "review"[Filter]

\#21 ((("risk factors"[Title/Abstract]) OR ("prevalence" OR "prevalence"[MeSH] )OR ("tuberculosis" OR "TB" OR "tuberculosis"[MeSH])) AND ("COPD" OR "obstructive lung disease")) AND ("Systematic Reviews as Topic"[MeSH] OR "review"[Publication Type] OR "review"[Filter] )

\#22 "humans"[Filter]

\#23 (("risk factors"[Title/Abstract]) OR ("prevalence" OR "prevalence"[MeSH]) OR ("tuberculosis" OR "TB" OR "tuberculosis"[MeSH] )) AND ("COPD" OR "obstructive lung disease")) AND ("humans"[Filter])

\#24 (("risk factors"[Title/Abstract]) OR ("prevalence" OR "prevalence"[MeSH]) OR ("tuberculosis" OR "TB" OR "tuberculosis"[MeSH])) AND ("COPD" OR "obstructive lung disease" )) AND ("humans"[Filter]) NOT("Systematic Reviews as Topic"[MeSH] OR "review"[Publication Type] OR "review"[Filter])
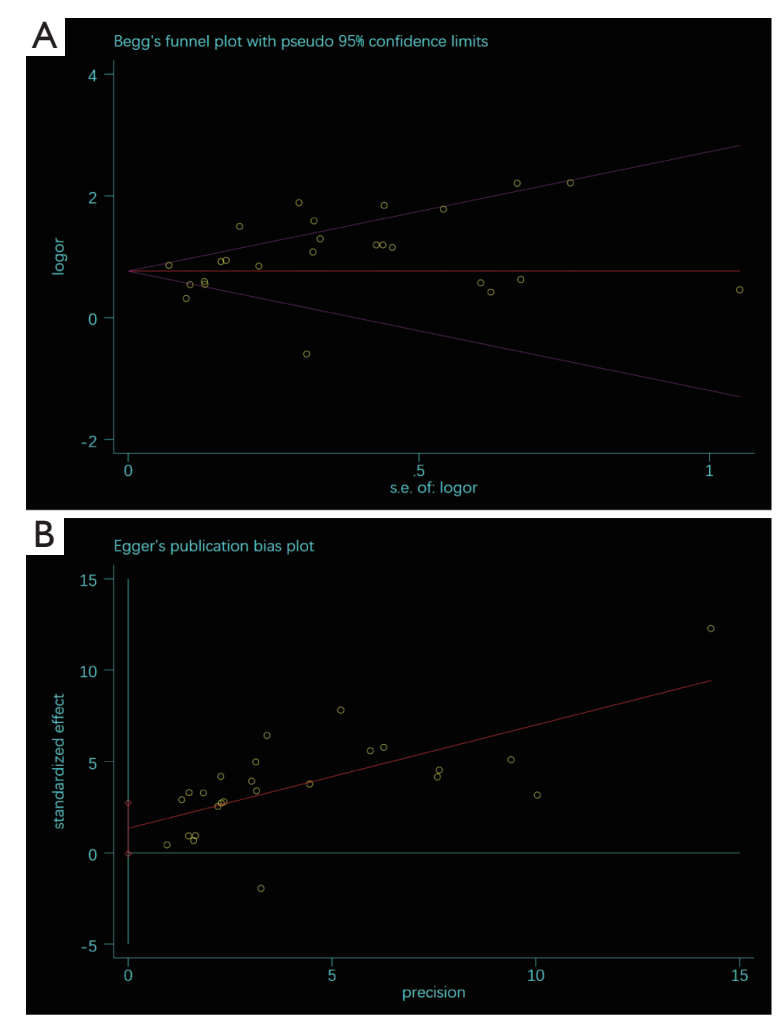

Figure S1 Funnel plots for (A) Begg's rank correlation test and (B) Egger’s linear regression. 